\title{
Modelling the Aerodynamics of Coaxial Helicopters - from an Isolated Rotor to a Complete Aircraft
}

\author{
Hyo Won Kim ${ }^{1}$, Richard E. Brown ${ }^{2}$
}

\begin{abstract}
This paper provides an overview of recent research on the aerodynamics of coaxial rotors at the Rotorcraft Aeromechanics Laboratory of the Glasgow University Rotorcraft Laboratories. The Laboratory's comprehensive rotorcraft code, known as the Vorticity Transport Model, has been used to study the aerodynamics of various coaxial rotor systems. Modelled coaxial rotor systems have ranged from a relatively simple twin two-bladed teetering configuration to a generic coaxial helicopter with a stiff main rotor system, a tail-mounted propulsor, and a horizontal stabiliser. Various studies have been performed to investigate the ability of the Vorticity Transport Model to reproduce the detailed effect of the rotor wake on the aerodynamics and performance of coaxial systems, and its ability to capture the aerodynamic interactions that arise between the various components of realistic, complex, coaxial helicopter configurations. It is suggested that the use of such a numerical technique not only allows insight into the performance of such rotor systems but might also eventually allow the various aeromechanical problems that often beset new helicopter designs of this type to be circumvented at an early stage in their design.
\end{abstract}

\section{Introduction}

The flow field around a helicopter has posed a significant modelling challenge to the computational fluid dynamics (CFD) community due to the dominant and persistent nature of the vortical structures that exist within the wake generated by its rotors. CFD schemes based on the traditional pressure-velocity formulation of the Navier-Stokes equations generally struggle to preserve these vortical structures as the vorticity in the flow is quickly diffused through numerical dissipation. The effect of the artificial viscosity that arises from numerical dissipation can be reduced by increasing the grid resolution but the computation soon becomes pro-

\footnotetext{
${ }^{1}$ Postgraduate Research Student, Imperial College London, UK (Currently at University of Glasgow as a Visiting Researcher) E-mail: hyo.kim04@imperial.ac.uk

${ }^{2}$ Mechan Chair of Engineering, University of Glasgow, UK
} 
hibitively expensive. Of course, the problem is exacerbated further when a full helicopter configuration is considered, especially where the interaction between two (or more) geometrically separated components via their wakes acts to modify their aerodynamic loading. The inability to predict the consequences of certain interactional aerodynamics has indeed led to unexpected flight mechanic issues in many prototype helicopters [1-6]. In several cases, such interactions have resulted in significant overrun of development costs.

Modern requirements for high performance call for a new generation of highly innovative rotorcraft that are capable of both heavy-lift and high speed. Several non-conventional helicopter configurations, such as the tilt rotor and compound helicopter, have been put forward as possible solutions to these requirements. One such proposal, Sikorsky Aircraft Corporation's X2 Technology Demonstrator [7], is based on a rigid coaxial rotor platform similar to the Advancing Blade Concept (ABC) rotor of the XH-59A, developed by the same company in the 1970s [8]. The advantage of a coaxial rotor with significant flapwise stiffness is that the effects of stall on the retreating blade can be delayed to higher forward flight speed as the laterally unbalanced load on one rotor can be compensated for by an equivalent, anti-symmetric loading on the other, contra-rotating rotor. The other limiting factor on the attainable speed, the effect of compressibility at the tip of the advancing blade, can be deferred by using an auxiliary device to augment the propulsive thrust of the main rotor. This allows the main rotor system to be offloaded, thus delaying the effects of compressibility to higher forward speed. In a system of such complexity, it is fair to expect the sub-components to interact aerodynamically, and hence their performance to be quite different when integrated as a complete rotorcraft compared to when analysed in isolation.

The aim of the studies surveyed in this paper was to demonstrate that the current state of the art in computational modelling of helicopter aerodynamics has progressed in recent years to the point where the interactive aerodynamic flow field associated with a coaxial rotor system, and hence its performance, can be captured accurately. This survey will demonstrate that high fidelity computational simulations are capable of lending a detailed insight into the interactive aerodynamic environment of a new rotorcraft, even one with as complex a configuration as that of the compounded coaxial helicopter. The hope is that such analyses may soon be integrated early in the development of all rotorcraft, where they might help to avoid some of the costly mistakes that have been committed during the design of this extremely complex type of flying machines in the past.

\section{Computational Model}

The VTM is a comprehensive code tailored for the aeromechanical analysis of rotorcraft systems. The model was initially developed by Brown [9] and later ex- 
tended by Brown and Line [10]. Unlike a conventional CFD approach, the governing flow equations are recast into vorticity-velocity form to yield

$$
\frac{\partial}{\partial t} \omega+u \cdot \nabla \omega-\omega \cdot \nabla u=v \nabla^{2} \omega
$$

This form of the Navier-Stokes equation allows vorticity to be conserved explicitly. The vorticity transport equation is discretised and solved using a finite volume TVD scheme which is particularly well suited to preserving the compactness of the vortical structures in the rotor wake for long periods of time. In the context of coaxial rotor aerodynamics, this property of the VTM enables the longrange aerodynamic interactions between the twin main rotors and any other geometrically well-separated components of the aircraft to be captured and resolved in detail. The flow is assumed to be inviscid everywhere except on the solid surfaces immersed in the flow. The generation of vorticity by lifting elements such as the rotor blades or fuselage appendages is then accounted for by treating these components as sources of vorticity, effectively replacing the viscous term in Equation (1) with a source term, $S$. The aerodynamics of the rotor blades are modelled using an extension of the Weissinger-L version of lifting-line theory in conjunction with a look-up table for the two-dimensional aerodynamic characteristics of the blade sections. The temporal and spatial variation of the bound vorticity, $\omega_{b}$, then yields the source term

$$
S=-\frac{d}{d t} \omega_{b}+u_{b} \nabla \cdot \omega_{b}
$$

The aerodynamics of the fuselage is modelled using a vortex panel approach in which the condition of zero through-flow is satisfied at the centroid of each panel. Lift generation by the fuselage is modelled by applying the Kutta condition along pre-specified separation lines on its surface. The viscous wake of the fuselage is not accounted for at present, however. The equations of motion for the blades, as forced by the aerodynamic loading along their span, are derived by numerical differentiation of a pre-specified non-linear Lagrangian for the particular system being modelled. No small-angle approximations are involved in this approach and the coupled flap-lag-feather dynamics of each of the blades are fully represented.

The acoustics, where applicable, are computed using the Farassat-1A formulation of the Ffowcs Williams-Hawkings equations. The aerodynamic force contribution from each collocation point along each blade is used to construct a set of point acoustic sources, integration of which over the span of the blades yields the loading noise contribution. The lifting-line approach to the blade aerodynamics assumes an infinitesimally thin blade and hence the thickness contribution to the noise is modelled using a source-sink pair attached to each blade panel. The noise contribution from quadrupole terms as well as that from the fuselage is neglected. 
The VTM has shown considerable success in both capturing and preserving the complex vortex structures that are contained within the wakes of conventional helicopter rotors $[9,10]$ and has been used previously to study rotor response to wake encounters $[11,12]$, the vortex ring state $[13,14]$, the influence of ground effect $[15,16]$, and the acoustics of a model rotor [17].

In this paper, a review of the study of coaxial rotor aerodynamics undertaken at the Rotorcraft Aeromechanics Laboratory of Glasgow University Rotorcraft Laboratories using the VTM is provided. The ability of the method to capture convincingly the aerodynamics of a coaxial rotor system in isolation and as a part of a more complex full helicopter system is demonstrated in the following sections of this paper.

\section{Aerodynamics of a Hinged Coaxial Rotor}

\section{1. Aerodynamic Performance of an Isolated Coaxial Rotor}

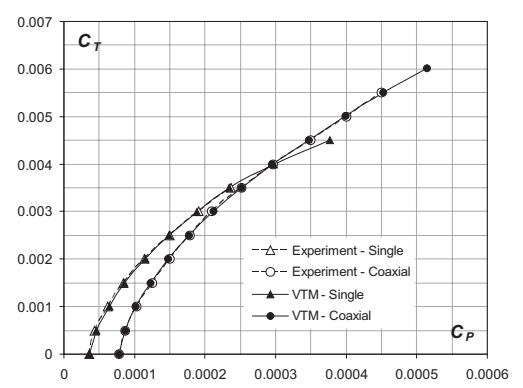

Fig. 1. Total power consumption $\left(C_{P}\right)$ as a function of thrust $\left(C_{T}\right)$ in hover - VTM simulations compared to Harrington's experiment [18].

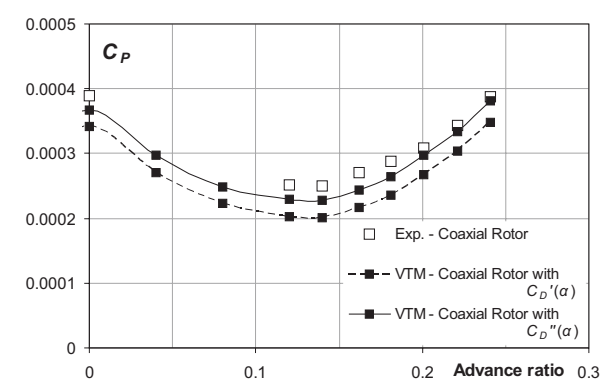

Fig. 2. Total power consumption $\left(C_{P}\right)$ in steady level flight as a function of forward speed (thrust coefficient $\left.C_{T}=0.0048\right)$ - Dingeldein's experiment compared to VTM simulations with two different drag polars, $C_{D}{ }^{\prime}(\alpha)$ and $C_{D}{ }^{\prime \prime}(\alpha)[18]$.

The reliability of the VTM's representation of coaxial rotor performance has been assessed in Ref. 18 by comparing the predicted power consumption of Harrington's coaxial rotor to that experimentally measured by Harrington [19] in hover and by Dingeldein [20] in forward flight (see Figs. 1 and 2). Comparison of the numerical predictions against the experimental data shows that the overall power consumption is particularly sensitive to the model that is used to represent the drag polar for the blade aerofoil sections, and possibly thus to the precise operating conditions of the rotor blade. However, some degree of absolute quantification does appear to be justified when this variability in profile drag, hence pro- 
file power, is removed to reveal the induced component of the power consumption, as in the comparisons presented in the next section of this paper.

\section{2. A Rational Approach for Comparing Coaxial and Single Rotors}

The relative merit of a twin coaxial rotor over a conventional single rotor in terms of efficiency and performance has long been a point of contention. Comparisons made in the existing literature have often failed to account correctly for the essential differences in the configurations of the two types of rotor and thus on occasion have drawn seemingly conflicting conclusions [21]. Numerical results from the VTM have been used to establish a rational approach to a like-for-like comparison of performance between coaxial and conventional single rotor systems [18]. It should be borne in mind, however, when extrapolating isolated rotor data to full helicopter systems, that the comparisons of performance can be skewed by the additional $5-10 \%$ of the main rotor power that is required by the tail rotor of the single rotor platform to trim the yaw moment in the system [22]. This torque compensation is provided inherently within the coaxial system.

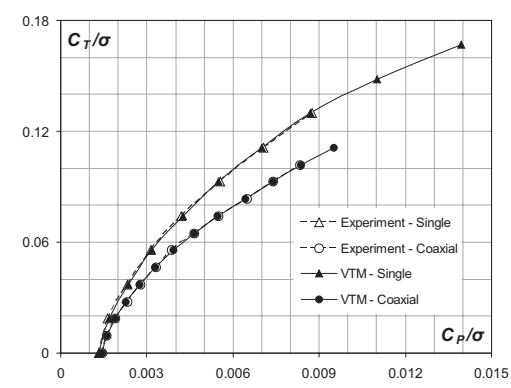

Fig. 3. Total power consumption $\left(C_{P}\right)$ as a function of thrust $\left(C_{T}\right)$ in hover - comparison of the coaxial rotor with one of its constituent rotors ('single') after normalising by solidity $\sigma$ [18].

The numerical results obtained by replicating Harrington's experiment have been used to highlight the potential for misrepresentation of the relative merit of the coaxial rotor when compared to a rotor of more conventional configuration. In the experiment, the performance of one of the constituent rotors of the coaxial system was compared to the performance of the entire coaxial system. Because of its lower solidity, the single rotor is inherently limited in thrust-generating capability by blade stall and hence, even when the numerical results are normalised by solidity, the comparison of the performance of the two systems is misleading (see Fig. 3). It was proposed that the equivalent, conventional single rotor should thus have the same total number of blades as the coaxial system and that the blades of the two systems should be geometrically identical. In Figs. 4 and 5, this definition is shown to yield a fair like-for-like comparison between the two disparate sys- 
tems. It is seen that the difference in performance of the two types of rotor is actually of the same order as the plausible variability in the profile power of the two systems. The two rotors have the same solidity and thus the lift potential of the two systems is matched. In other words, blade stall cannot obscure the comparison between the two systems. The blades also operate in a comparable aerodynamic environment. The differences in the performance of the two systems are thus induced solely by the fundamental difference in the way that the wake of the two types of rotor interacts with the blades. Definition of the equivalent conventional rotor in this manner thus yields a rational approach to comparing the relative performance of coaxial and single rotor systems.

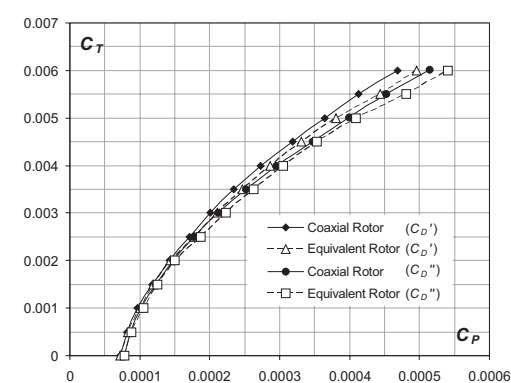

Fig. 4. Total power consumption $\left(C_{P}\right)$ as a function of thrust $\left(C_{T}\right)$ in hover - comparison between rotors with identical solidity and blade properties [18].

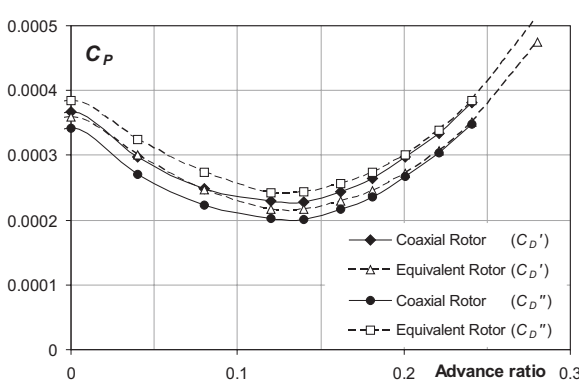

Fig. 5. Total power consumption $\left(C_{P}\right)$ in steady level flight as a function of forward speed (thrust coefficient $\left.C_{T}=0.0048\right)-$ comparison between rotors with identical solidity and blade properties [18].

\section{3. Comparison of Performance in Steady and Manoeuvring Flight}

The performance of a coaxial rotor in hover, in steady forward flight, and in a level, coordinated turn has been contrasted with that of an equivalent, conventional rotor defined, as motivated in the previous section, as a single, conventional rotor with the same overall solidity, number of blades and blade aerodynamic properties [18]. Simulations using the VTM have allowed the differences in the performance of the two systems (without undue complication from fuselage and tail rotor effects) to be investigated in terms of the profile, induced and parasite contributions to the overall power consumed by the rotors (see Figs. 6 to 8), and to be traced to the differences in the structure of the wakes of the two systems.

In hover, the coaxial system consumes less induced power than the equivalent, conventional system. The wake of the coaxial system in hover is dominated, close to the rotors, by the behaviour of the individual tip vortices from the two rotors as they convect along the surface of two roughly concentric, but distinct, wake tubes. The axial convection rate of the tip vortices, particularly those from the upper ro- 
tor, is significantly greater than for the tip vortices of the same rotor operating in isolation. The resultant weakening of the blade-wake interaction yields significantly reduced induced power consumption on the outer parts of the upper rotor that translates into the observed benefit in terms of the overall induced power required by the coaxial system.

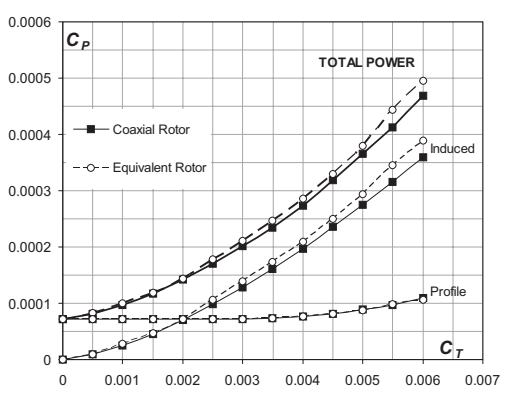

Fig. 6. Total power consumption, together with its constituents, in hover as a function of thrust - comparison of the coaxial rotor with the equivalent single rotor [18].

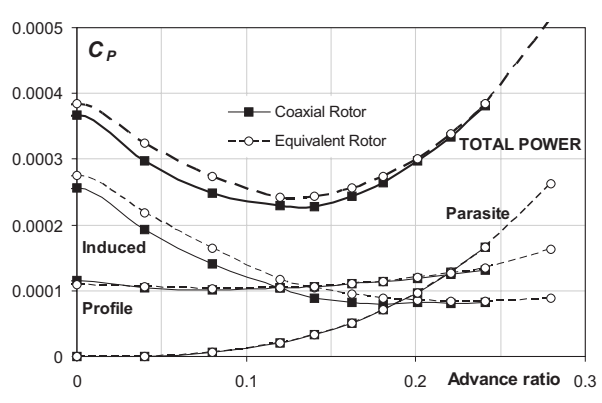

Fig. 7. Total power consumption, together with its constituents, in steady level flight as a function of forward speed (thrust coefficient $C_{T}=0.0048$ ) [18].

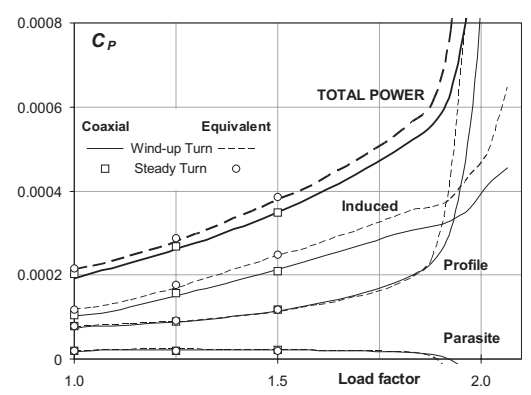

Fig. 8. Total power consumption, together with its constituents, in a level, wind-up turn as a function of the load factor of the turn [18].

In steady forward flight, the coaxial rotor again shows a distinct induced power advantage over its equivalent, conventional system at transitional and low advance ratios, but at high advance ratio there is very little difference between the performance of the two systems. At a thrust coefficient, $C_{T}$, of 0.0048 , the maximum forward flight speed of the systems that were simulated was limited to an advance ratio of about 0.28 by stall on the retreating side of the rotors. The rather limited maximum performance of the two systems was most likely related to their low solidity. With the coaxial system, the near-simultaneous stall on the retreating sides of both upper and lower rotors leads to backwards flapping of both discs, although blade strike occurs at the back of the system because the upper rotor stalls more severely than the lower. 
The structure of the wake generated by the coaxial and conventional systems is superficially similar at all advance ratios, and shows a transition from a tube-like geometry at low advance ratio to a flattened aeroplane-like form as the advance ratio is increased ${ }^{3}$ (see Fig. 9). The formation of the wake of the coaxial rotor at post-transitional advance ratio involves an intricate process whereby the vortices from both upper and lower rotors wind around each other to create a single, merged pair of super-vortices downstream of the system (see Fig. 9). The loading on the lower rotor is strongly influenced by interaction with the wake from the upper rotor, and there is also evidence on both rotors of intra-rotor wake interaction especially at low advance ratio. In comparison, the inflow distribution on the conventional rotor, since the inter-rotor blade-vortex interactions are absent, is very much simpler in structure.

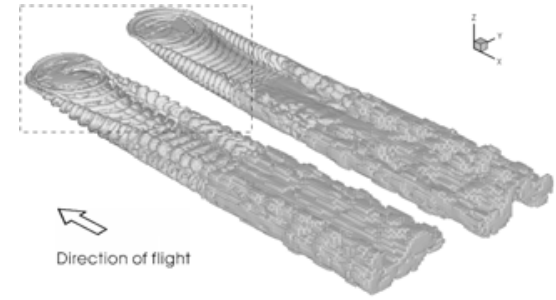

(a) Overall wake geometry

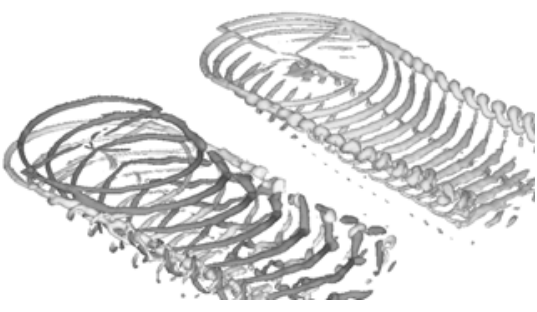

(b) Tip vortex geometry (coaxial rotor: upper rotor vortices shaded darker than lower)

Fig. 9. Wake structure of coaxial rotor (left) and equivalent single rotor (right) in forward flight at advance ratio $\mu=0.12$.

Simulations of a wind-up turn at constant advance ratio again show the coaxial rotor to possess a distinct advantage over the conventional system (see Fig. 8) - a reduction in power of about $8 \%$ for load factors between 1.0 and 1.7 is observed at an advance ratio of 0.12 and a thrust coefficient of 0.0048 . As in forward flight, the improved performance of the coaxial rotor results completely from a reduction in the induced power required by the system relative to the conventional rotor. This advantage is offset to a certain degree by the enhanced vibration of the coaxial system during the turn compared to the conventional system. As in steady level flight, the turn performance is limited by stall and, in the coaxial system, by subsequent blade strike, at a load factor of about 1.7 for the low-solidity rotors that were used in this study. The inflow distribution on the rotors is subtly different to that in steady, level flight, and a progressive rearwards shift in the positions of the interactions between the blades and their vortices with increasing load factor appears to be induced principally by the effects of the curvature of the trajectory on the geometry of the wake.

The observed differences in induced power required by the coaxial system and the equivalent, conventional rotor originate in subtle differences in the loading dis-

\footnotetext{
${ }^{3}$ The transition occurs at an advance ratio of approximately 0.1 .
} 
tribution on the two systems that are primarily associated with the pattern of blade-vortex interactions on the rotors. The beneficial properties of the coaxial rotor in forward flight and in steady turns appear to be a consequence of the somewhat greater lateral symmetry of its loading compared to the conventional system. This symmetry allows the coaxial configuration to avoid, to a small extent, the drag penalty associated with the high loading on the retreating side of the conventional rotor. It is important, though, to acknowledge the subtlety of the effects that lead to the reduced induced power requirement of the coaxial system, and thus the rather stringent requirements that are imposed on the fidelity of numerical models in order for these effects to be resolved.

\section{Aerodynamics of a Stiffened Hingeless Coaxial Rotor}

The effects of flapwise stiffness on the performance of a coaxial rotor were studied using a modified form of Harrington's rotor as described in Ref. 23. The effects of hub stiffness on the natural frequency of blade flapping are introduced into the simulations by modelling the blades of the rotors as being completely rigid, but then applying a spring across each flapping hinge of their articulated hubs.

Introduction of flapwise stiffness into the system has a marked effect on the power consumption of the coaxial rotor in forward flight. VTM calculations suggest that the equivalent articulated system has a power requirement that is over twenty percent greater than that of a completely rigid rotor system when trimmed to an equivalent flight condition. Most of this enhanced power requirement can be attributed to a large increase in the induced power that is consumed by the system when the blades of the rotors are allowed to flap freely. Most of the advantage of the rigid configuration is retained if the stiffness of the rotors is reduced to practical levels, but the advantage of the system with finite stiffness over the conventional articulated system deteriorates quite significantly as the forward speed of the system is reduced. In high-speed forward flight, significant further power savings can be achieved, at least in principle, if an auxiliary device is used to alleviate the requirement for the main rotor system to produce a propulsive force component, and, indeed, such an arrangement might be necessary to prevent rotor performance being limited by aerodynamic stall.

Simulations suggest that the unsteady aerodynamic forcing of very stiff rotor systems is relatively insensitive to the actual flapwise stiffness of the system. This implies that simple palliative measures such as structural tailoring may have little effect in being able to modify the inherent vibrational characteristics of stiff coaxial rotor systems. The principal effect of the aerodynamic forcing of very stiff coaxial systems is to produce an excitation of the system, primarily at the fundamental blade passing frequency, in both pitch and heave, but aerodynamic interference 
between the rotors may introduce a small component of roll excitation into the vibration of the rotor.

The numerical results presented in Ref. 23 lend strong support to the existing contention that the introduction of flapwise stiffness can lead to a coaxial rotor system that possesses clear advantages in performance over the corresponding articulated system, and hence, by comparison against the results of previous studies [18], over the equivalent, conventional, planar rotor system. The results also lend some support, though, to the contention that the advantages of the coaxial rotor configuration in terms of overall performance may need to be offset against fundamentally unavoidable penalties in terms of the vibration, and thus, possibly, the noise that is produced by the lower rotor as a result of its aerodynamic interference with the wake that is produced by the upper rotor. It seems risky to jump to such overarching conclusions in the absence of the further insight that could be gleaned from simulations of rotor systems that are more physically representative of full-scale practice than those tested in Ref. 23, however.

\section{Thrust-Compounded Coaxial Helicopter}

The thrust-compounded coaxial helicopter with stiffened rotors is a particularly plausible contender to meet modern requirements for a new generation of high performance rotorcraft. The VTM has been used to study the aerodynamic interactions between the sub-components of a generic, but representative helicopter with this configuration. These studies [24, 25] are summarised below.

\section{1. Helicopter Model}

The generic helicopter configuration studied in Refs. 24 and 25 comprises a stiffened contra-rotating coaxial rotor system, a tail-mounted propulsor, and a streamlined fuselage featuring a horizontal tailplane at its rear (see Fig. 10). In the interests of brevity, only a brief description of the configuration is presented here but a more detailed geometric description can be found in Kim et al. [24].

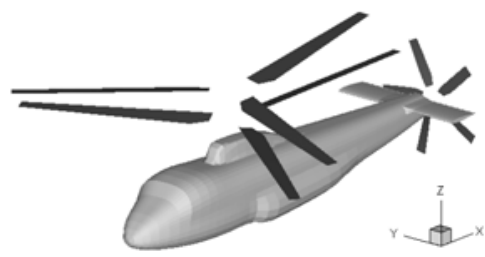

Fig. 10. Generic thrust-compounded hingeless coaxial helicopter configuration [24, 25]. 
The coaxial system consists of two three-bladed rotors. The stiffness in the rotor system is approximated, somewhat crudely but supported by the results contained in Ref. 23, by assuming the rotor blades and their attachments to the hub to be completely rigid. The propulsor is a five-bladed variable pitch propeller mounted in a pusher configuration to provide auxiliary propulsive thrust, offloading the main rotor in high speed forward flight. The geometry of the fuselage is entirely fictitious but the compact and streamlined design is chosen to be representative of a realistic modern helicopter with high speed requirements. In line with current design practice to yield sufficient longitudinal stability and control, a rectangular tailplane, just forward of the propulsor, is also incorporated into the design. This configuration was developed specifically to provide a realistic representation of the aerodynamic interactions that might occur in practical semi-rigid, thrust-compounded coaxial helicopter systems.

\section{2. Interactional Aerodynamics and Aeroacoustics}

The aerodynamic environment of the configuration described above is characterised by very strong aerodynamic interactions between its various components (see Fig. 11).

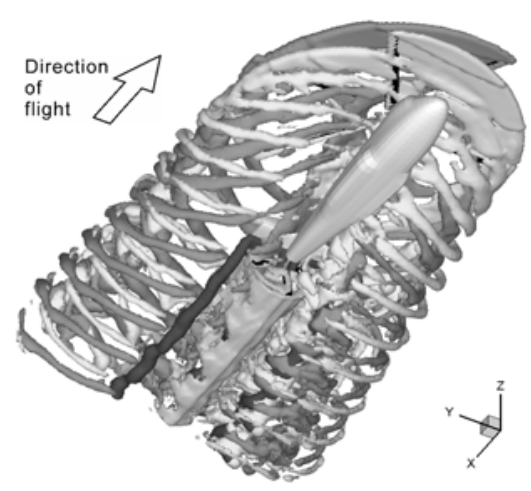

(a) Bottom view

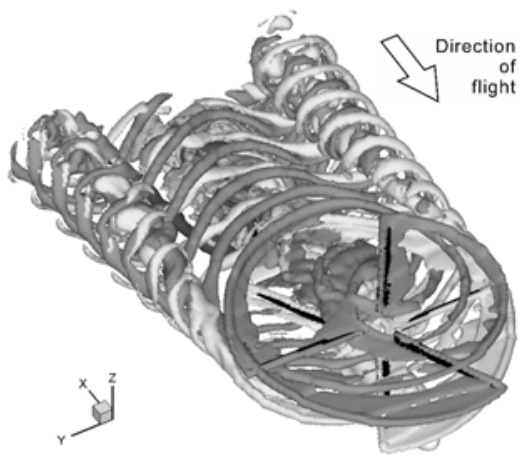

(b) Top view

Fig. 11. Visualisation of the wake structure of the thrust-compounded coaxial helicopter using surface contours of constant vorticity at advance ratio $\mu=0.15$ [25].

The aerodynamic environment of the main rotors of the system is dominated by the direct impingement of the wake from the upper rotor onto the blades of the lower rotor, and, particularly at low forward speed, the thrust and torque produced by the system are highly unsteady. The fluctuations in the loading on the coaxial system occur primarily at the fundamental blade-passage frequency and are particularly strong as a result of the phase relationship between the loading on the upper and lower rotors. A contribution to the loading on the coaxial rotor at twice the 
blade-passage frequency is a result of the loading fluctuations that are induced on the individual rotors of the system as a result of direct blade overpassage.

As shown in Fig. 12, the wake of the main rotor sweeps over the fuselage and tailplane at low forward speed, inducing a significant nose-up pitching moment on the tailplane that must be counteracted by longitudinal cyclic input to the main rotor. This pitch-up characteristic has been encountered during the development of several helicopters and has proved on occasion to be very troublesome to eradicate. Over a broad range of forward flight speeds, the wake from the main rotor is ingested directly into the propulsor, where it induces strong fluctuations in the loading produced by this rotor. These fluctuations occur at both the blade-passage frequency of the main rotor and of the propulsor, and have the potential to excite significant vibration of the aircraft. VTM calculations suggest that this interaction, together with poor scheduling of the partition of the propulsive thrust between the main rotor and the rear-mounted propulsor with forward speed, can lead to a distinctly non-optimal situation where the propulsor produces significant vibratory excitation of the system but little useful contribution to its propulsion.

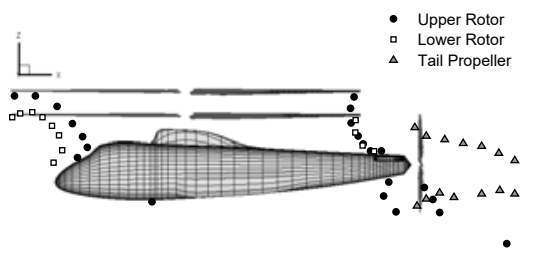

(a) $\mu=0.05$

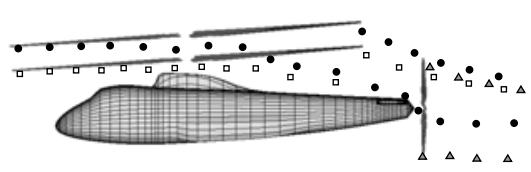

(c) $\mu=0.15$

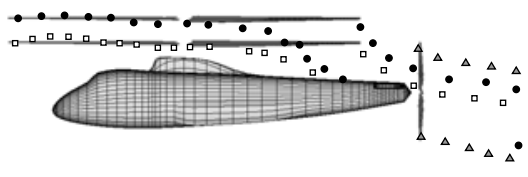

(b) $\mu=0.10$

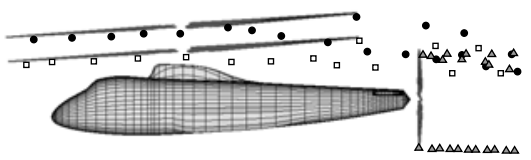

(d) $\mu=0.30$

Fig. 12. Trajectories of the tip vortices of the main rotors and propulsor where they intersect the vertical plane through the fuselage centreline [24].

The propulsor also induces significant vibratory forcing on the tailplane at high forward speed. This forcing is at the fundamental blade-passage frequency of the propulsor, and suggests that the tailplane position in any vehicle where the lifting surface and propulsor are as closely coupled as in the present configuration may have to be considered carefully to avoid shortening its fatigue life. Nevertheless, the unsteady forcing of the tailplane is dominated by interactions with the wake from the main rotor - the fluctuations at the fundamental blade passage frequency that were observed in the pressure distribution on the tailplane are characteristic of the close passage of individual vortices over its surface. 
Finally, predictions of the acoustic signature of the system presented in Refs. 24 and 25 suggest that the overall noise produced by the system, at least in slow forward flight, is significantly higher than that produced by similar conventional helicopters in the same weight class. The major contribution to the noise produced by the system in the highly objectionable BVI frequency range comes from the lower rotor because of strong aerodynamic interaction with the upper rotor. The propulsor contributes significant noise over a broad frequency spectrum. At the flight condition that was considered, much of this noise is induced by interactions between the blades of the propulsor and the wake of the main rotor and tailplane.

The numerical calculations were thus able to reveal many of the aerodynamic interactions that might be expected to arise in a configuration as aerodynamically complex as the generic thrust-augmented coaxial helicopter that formed the basis of the study. It should be acknowledged though that the exact form, and particularly the effect on the loading produced on the system, of these interactions would of course vary depending on the specifics of the configuration, and that many of the pathological conditions exposed in the study could quite feasibly have been rectified by careful aerodynamic re-design.

\section{3. Understanding the Interactions}

By comparing the aerodynamics of the full configuration of the helicopter to the aerodynamics of various combinations of its sub-components, the influence of the various aerodynamic interactions within the system on its behaviour could be isolated as described in Ref. 25.

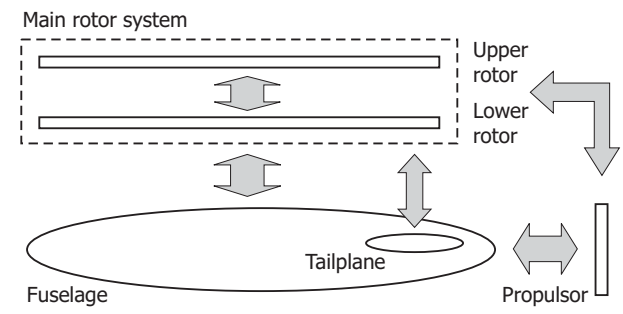

Fig. 13. Schematic summarising the network of aerodynamic interactions between various components of the simulated configuration [25].

The traditional approach to the analysis of interactional effects on the performance of a helicopter relies on an initial characterisation of the system in terms of a network of possible interactions between the separate components of its configuration (see Fig. 13). Thus, within the configuration that was studied in Ref. 25, it is possible to identify the effect of the main rotor on the fuselage and propulsor, the distortion of the wake of the main rotor that is caused by the presence of the fuse- 
lage and so on. The characteristics of these various interactions and their effects on the performance of the system have been described in detail in Ref. 25.

Many of the interactions that were exposed within the aerodynamics of the configuration have exhibited a relatively linear relationship between cause and effect and hence would be amenable to the reductionist approach described above. For instance, the distortion of the wake of the main rotor by the fuselage has a marked effect on the loading generated by the propulsor, but the effect on the propulsor is prevented from feeding back into the performance of the main rotor. This is because of the isolation that is provided by the particular method that was used to trim the vehicle, and also by the inherent directionality of the interaction that results from its physics being dominated by the convection of the wakes of the two systems into the flow behind the vehicle.

Several of the interactions that were observed for this helicopter configuration exhibited a less direct relationship between cause and effect, however. These interactions are characterised by strong feedback or closed-loop type behaviour, in certain cases through a path which remains relatively obscure and hidden within the network of interactions that form the basis of the traditional reductionist type approach. For instance, the load that is induced on the tailplane by the direct impingement of the wake of the main rotor requires, through the requirement for overall trim of the forces and moments on the aircraft, a compensatory change in the loading distribution on the main rotor itself, which then modifies the strength of its wake and hence, in circular fashion, the loading on the tailplane itself. Without this understanding of the strong mutual coupling between the performance of the tailplane and the main rotor, the observed dependence of the acoustic radiation of the aircraft on the presence or not of the tailplane (or, in practical terms, more likely on its design and positioning) may appear to the analyst as a very obscure and possibly even unfathomable interdependence within the system.

Thus, although the reductionist, network-based approach to classifying the interactions present within the system is conceptually appealing and simple, it must be realised that the possible presence of feedback loops deep within the interactional aerodynamics, such as the one described above, may cause the approach to miss, obscure or hide the presence of interactions between some of the various sub-components of the system. The analysis presented in Ref. 25 warns against an overly literal application of this reductive, building-block type approach to the categorisation of the interactions that are present within the system.

\section{Conclusion}

The results of a programme of computational study of coaxial rotor aerodynamics conducted using the Vorticity Transport Model at the Rotorcraft Aeromechanics Laboratory of the Glasgow University Rotorcraft Laboratories have been summarised. 
Analysis of the computational results obtained using the VTM suggests that the differences in performance between a helicopter with a coaxial rotor system and the equivalently defined system with a conventional, single rotor are subtle, and generally result from small differences in the character and strength of the localised interaction between the blades of the rotors and the wakes that they produce. Reliable prediction of these effects is well beyond the scope of simple models and is absolutely dependent on accurate prediction of the detailed structure of the rotor wake.

The study lends weight to the assertion though that the state of the art of computational helicopter aerodynamic predictions is advancing to a stage where the use of powerful models such as the VTM may allow useful insight into the likely aeromechanical behaviour of realistic helicopter configurations. Furthermore, it is suggested that there may be no real substitute for detailed simulations of the entire configuration if the effects on the performance of the vehicle of the most deeply hidden interactions within the system are to be exposed. It has been shown that modern numerical techniques are indeed capable of representing the very wide range of aerodynamic interactions that are present within the helicopter system, even one as complex as a compounded coaxial system. This bodes well for the assertion that modern computational techniques may be in a position to help circumvent future repetition of the long history of unforeseen, interaction-induced dynamic problems that have manifested on prototype or production aircraft.

\section{References}

[1] Cooper, D.E., "YUH-60A Stability and Control," Journal of the American Helicopter Society, Vol. 23, No. 3, 1978, pp. 2-9.

[2] Prouty, R.W., Amer, K.B., "The YAH-64 Empennage and Tail Rotor - A Technical History," American Helicopter Society $38^{\text {th }}$ Annual Forum Proceedings, Anaheim, CA, 4-7 May 1982, pp. 247-261.

[3] Main, B.J., Mussi, F., "EH101 - Development Status Report," Proceedings of the $16^{\text {th }}$ European Rotorcraft Forum, Glasgow, UK, 18-20 September 1990, pp. III.2.1.1-12.

[4] Cassier, A., Weneckers, R., Pouradier, J., "Aerodynamic Development of the Tiger Helicopter," Proceedings of the American Helicopter Society $50^{\text {th }}$ Annual Forum, Washington DC, 11-13 May 1994.

[5] Eglin, P., "Aerodynamic Design of the NH90 Helicopter Stabilizer," Proceedings of the $23^{\text {rd }}$ European Rotorcraft Forum, Dresden, Germany, 16-18 September 1997, pp. 68.1-10.

[6] Frederickson, K.C., Lamb, J.R., "Experimental Investigation of Main Rotor Wake Induced Empennage Vibratory Airloads for the RAH-66 Comanche Helicopter," Proceedings of the American Helicopter Society $49^{\text {th }}$ Annual Forum, St. Louis, MO, 19-21 May 1993, pp. 10291039.

[7] Bagai, A., "Aerodynamic Design of the Sikorsky X2 Technology Demonstrator ${ }^{\mathrm{TM}}$ Main Rotor Blade," American Helicopter Society $64^{\text {th }}$ Annual Forum Proceedings, Montréal, Canada, 29 April-1 May 2008.

[8] Burgess, R.K., "The ABC ${ }^{\mathrm{TM}}$ Rotor - A Historical Perspective," American Helicopter Society $60^{\text {th }}$ Annual Forum Proceedings, Baltimore, MD, 7-10 June 2004. 
[9] Brown, R.E., "Rotor Wake Modeling for Flight Dynamic Simulation of Helicopters," AIAA Journal, Vol. 38, No. 1, January 2000, pp. 57-63.

[10] Brown, R.E., Line, A.J., "Efficient High-Resolution Wake Modeling Using the Vorticity Transport Equation," AIAA Journal, Vol. 43, No. 7, April 2005, pp. 1434-1443.

[11] Whitehouse, G.R., Brown, R.E., "Modeling the Mutual Distortions of Interacting Helicopter and Aircraft Wakes," AIAA Journal of Aircraft, Vol. 40, No. 3, May 2003. pp. 440-449.

[12] Whitehouse, G.R., Brown, R.E., "Modelling a Helicopter Rotor's Response to Wake Encounters," Aeronautical Journal, Vol. 108, No. 1079, January 2004. pp. 15-26.

[13] Ahlin, G.A., Brown, R.E., "Investigating the Physics of Rotor Vortex-Ring State using the Vorticity Transport Model," Paper 89, 31 $1^{\text {st }}$ European Rotorcraft Forum, Florence, Italy, 13-15 September 2005.

[14] Ahlin, G.A., Brown, R.E., "The Vortex Dynamics of the Rotor Vortex Ring Phenomenon," American Helicopter Society $63^{\text {rd }}$ Annual Forum Proceedings, Virginia Beach, VA, 1-3 May 2007.

[15] Brown, R.E., Whitehouse, G.R., "Modeling Rotor Wakes in Ground Effect," Journal of the American Helicopter Society, Vol. 49, No. 3, July 2004. pp. 238-249

[16] Phillips, C., Brown, R.E., "Eulerian Simulation of the Fluid Dynamics of Helicopter Brownout," American Helicopter Society $64^{\text {th }}$ Annual Forum Proceedings, Montréal, 29 April-1 May 2008

[17] Kelly, M.E., Duraisamy, K., Brown, R.E., "Blade Vortex Interaction and Airload Prediction using the Vorticity Transport Model," American Helicopter Society Specialists' Conference on Aeromechanics, San Francisco, CA, 23-25 January 2008.

[18] Kim, H.W., Brown, R.E., "Coaxial Rotor Performance and Wake Dynamics in Steady and Manoeuvring Flight," American Helicopter Society $62^{\text {nd }}$ Annual Forum Proceedings, Phoenix, AZ, 9-11 May 2006.

[19] Harrington, R.D., "Full-Scale-Tunnel Investigation of the Static-Thrust Performance of a Coaxial Helicopter Rotor," NACA TN-2318, March 1951.

[20] Dingeldein, R.C., "Wind-Tunnel Studies of the Performance of Multirotor Configurations," NACA TN-3236, August 1954.

[21] Coleman, C.P., "A Survey of Theoretical and Experimental Coaxial Rotor Aerodynamic Research," NASA TP-3675, March 1997.

[22] Leishman, J.G., Principles of Helicopter Aerodynamics, Second Edition, Cambridge University Press, Cambridge, UK, 2006.

[23] Kim, H.W., Brown, R.E., "Impact of Trim Strategy and Rotor Stiffness on Coaxial Rotor Performance," $1^{\text {st }}$ AHS/KSASS International Forum on Rotorcraft Multidisciplinary Technology, Seoul, Korea, 15-17 October 2007.

[24] Kim, H.W., Kenyon, A.R., Duraisamy, K., Brown, R.E., "Interactional Aerodynamics and Acoustics of a Propeller-Augmented Compound Coaxial Helicopter," American Helicopter Society Aeromechanics Specialists' Meeting, San Francisco, CA, 23-25 January 2008.

[25] Kim, H.W., Kenyon, A.R., Duraisamy, K., Brown, R.E., "Interactional Aerodynamics and Acoustics of a Hingeless Coaxial Helicopter with an Auxiliary Propeller in Forward Flight," International Powered Lift Conference, London, UK, 22-24 July 2008. 